\title{
Contents, Vol. 75, 1931
}

Inhaltsverzeichnis.

Eigenarbeiten. Seite

Battels, M., Einfluß von vielfachen druckentlastenden Operationen bei Hydrophthalmus congenitus und gleichzeitige Entstehung von hoher Myopie ... 17

,-- , Habituelle und spontane Linsenluxation ... 20

,-- , Gibt es besondere Pupillenfasern für die indirekte

Reaktion? 22

,,-- Zur Arbeit von Birch-Hirschfeld: Ein Vergleichder Resultate der Altersstaroperation mit und ohnevorbereitetem Bindehautlappen 166

Behr, C, Ein weiterer Beitrag zur Anatomie und zurPathogenese der scheibenförmigen

Degeneration amhinteren Augenpol 216

Blaicknßr, ]., Zur Klinik und Behandlung des Ulcus serpens 304

Bormacher, H., Zur Röntgenologie des Foramen opticum 27

Burk, A., Zur Anatomie der Arteria hyaloidea persistens 168

Daniels, B., Untersuchungen zur Jodbehandlung der

Katarakta senilis $\quad 129$

Elkes, G., Beitrag zur Atiologie der Neuritis retrobulbaris 271

Elschnig, A., Altersstarextraktion in der Kapsel .... 1

Falía, M. $\uparrow$ Bemerkungen zu der Arbeit von Prof. Birch-Hirschfeld: Ein Vergleich der Resultate der Altersstaroperation usw 95

Fischer, F., Schwangerschaftsveränderung der Hypophyse

als Ursache bitemporaler Hemianopsie 343

Haas, F., Ein Verfahren zur zahlenmäßigen Bewertung

des Lichtsinns 69

Hegner, C. A., Eine erfolgreiche Starextraktion durch Kuh-

hornstoß 55

Hoffmann, W., Der Wert der Röntgenuntersuchung bei

Erkrankungen der Augenhöhle 243

Jaensch,P. A., Doppelseitige Trochlearisparese als einzige

Motilitätsstörung bei Schilddrüsentumor 58

Junius, P., Kurze Bemerkung zu derPublikation von Prof.A. Peters-Rostock: Die Lehre vom

Trachom einst undjetzt 11

Krassò, /.,KlinischeundhistologischeBeiträgezurKenntnis

der Tiefenwirkung von Buckys Grenzstrahlen am Auge 31

,-- , Grenzstrahlentherapie der Conjunctivitis acuta

und chronica catarrhalis 285

Kreibig, W., Über einen Fall von metastatischem Hyper-nephrom des Auges nebst Bemerkungen

über eine ungewöhnliche Form der Netzhautablösung . . . 326

Kunz, E., Sarkom der Iris 
Lämmerhirt, F. G. siehe Wegner, W.

Less-Simkovits, M. $\uparrow$ Zur Kasuistik der Veränderungen am

Bulbus durch Druck von Orbitaltumoren .... 264

Meller, /., Über die Schaffung von flächenhaften Ver-lötungen derNetzhaut mit der Aderhaut durch Endo-thermie 207

- IV -

Seite

Müller, E., Zum Problem der Amblyopie ohne Spiegel-

befund und des Schielens $\quad 354$

Ohm, J., Der optokinetische Nystagmus im Dienste der Praxis 146 Pascal, J. J., Die Skiaskopie mit dem Photoskop ... 88 Remky,E., Zur Osteomyelitis desOberkiefersbeimSäugling 240 Sattler, C. H., Über Operationen an den Tränenröhrchen 237 Schneider, R., Naevus vasculosus der Konjunktiva und

Episklera 136

,,-- Knötchenförmige Konjunktivitis durch Fettimpräg-

nation (Elschnig) 369

Simson, J., Eine Beobachtung von Kalkablagerungen im

Plexus chorioideus bei Retinitis pigmentosa . . . 164 Stewens, /., Progredienter Exophthalmus

nach Basedow-

operation. Über Beziehungen zwischen Thyreoidea

und Hypophyse 137

Vormann, J., Ein Fall von Wanderlinse als Folgezustand

einer kongenitalen Linsenektopie 160

Wegner, W. und F. G. Lämmerhirt, Plethysmographische

Untersuchungen über den Bulbuspuls $\quad 317$

Aus der Praxis îür die Praxis.

Großschop†, E. v., Schnellreifung des grauen Stars 371

Lautetstein, M., 1. Einiges über die medikamentöse und operative Behandîung des Pannus

trachomatosus. 2. Einiges über die operative Behandîung bei Entropium und Trichiasis ... 177

Reitsch, W., Heftpflaster-Schienenverband bei Entropium spasticum.- Zur Enukleationstechnik.

- Über Verwendung teilmat-tierter Gläser. - Heftpflasterstreifen zum Fixieren der Cha-

lazionpinzette $\quad 96$

Schmidt, L., Drehbare Zweistärkengläser 175

Cresellschaîtsberichte.

Ophthalmologische Gesellschaft in Wien. Sitzung vom 20. IV.,

18. V. und 15.VI. 1931

97

Bericht über die 59. Versammlung des Vereins rheinisch-west-fälischer Augenärzte am 8. März im Haus der ärztlichen Fort-bildung in Essen. .... 185

XX. Sitzung der Opbthalmologischen Abteilung der Gesellschaftfür Wissenschaît und Leben im rheinisch-westfälischen In-dustriebezirk am 24. Januar 1931 . . 106

Ungarische ophthalmologische Gesellschaft in Budapest. Sitzung

vom 5. XII. 1930 und 30. I. 1931

Sitzung am 27. März 1931 ... 193

American Ophthalmological Society. 67. Tagung, 1.-3. Juni,

Asheville, North Carolina 373 
Schweizerische Ophthalmologische Gesellschaft. 24. Jahresversammlung vom 13.-14. Juni 1931 in St. Gallen 385

Vereinigte Medizinische Gesellschaft in Kiew. Sektion für Augen-

heilkunde. Sitzung vom 5. und 21. Mai 1930119

Sitzungen vom 25. November und 14. Dezember $1930 \ldots 196$

Diagnose und Therapie $\quad 120,198,279,398$

Unfall- und Versicherungskunde 125, 203, 281

Blindenwesen 204, 283, 401

Buchbesprechungen 205

Personalien 206, 284

Tagesnachrichten $\quad 128$

Druckfehlerberichtigung 120, 206, 284

S. Sachregister 402

Namenregister $\quad 407$ 